grating) would give instead the dotted curve of Fig. 2. Moreover, for a singlet the central maximum of the $I_{0 \mathrm{nin}}$ curve is replaced by a deep minimum. It is evident, therefore, that the method of using interference between different parts of the diffraction image increases the resolution. When making this calculation the intensity distribution within the diffraction image has been assumed to follow a $(\sin x / x)^{2}$ curve, where $x$ is the distance from the centre of the image.

Experiments have been made by using artificial spectral lines made up by two slits polarized in perpendicular directions. The phenomena with the two minima in Fig. 2 for the $I_{0 \mathrm{~min}}$ curve are clearly revealed in these experiments. So far the instrumental arrangement has made necessary the use of optical systems of the focal ratio $f / 300$ or similar. In our instrument the separation of the beams is one minute of arc only.

Instead of examining the function $I_{0 \mathrm{~m} \ln }\left(\lambda_{0}\right)$ it may be preferable to measure the degree of visibility ${ }^{3}$ or only $I_{0 \max }\left(\lambda_{0}\right)-I_{0 \min }\left(\lambda_{0}\right)$. In the latter case when assuming diffraction according to a $(\sin x / x)^{2}$ function only, we find:

$$
\begin{aligned}
I_{0 \max }\left(\lambda_{0}\right)-I_{0 \min }\left(\lambda_{0}\right)= & \\
4 \int_{-\infty}^{+\infty} J(\lambda) \frac{\sin \left(\lambda-\lambda_{0}+\frac{d}{2}\right)}{\lambda-\lambda_{0}+\frac{d}{2}} & \cdot \frac{\sin \left(\lambda-\lambda_{0}-\frac{d}{2}\right)}{\lambda-\lambda_{0}-\frac{d}{2}} \mathrm{~d} \lambda
\end{aligned}
$$

In this formula the size of the diffraction image produced by the (rectangular) aperture of the spectrograph equals $\pi$.

In Anacapri, we have recently performed some successful experiments where the slit $S_{2}$ and photo tube $P h$ of Fig. 1 have been replaced by a photographic film. By photographing the solar spectrum in this way, about $6000 \AA$ (with our Babcock grating in fourth order) interference phenomena have been clearly traced in the Fraunhofer lines. In our first experiment the displace. ment of the two superimposed spectra has been $0.10 \mathrm{~mm}$. The diffraction image of the spectrograph (rectangular aperture) has been $0.12 \mathrm{~mm}$ and the image of $S_{1}$ on the photographic film $0.03 \mathrm{~mm}$. As can be inferred from Fig. 3, fairly strong interference fringes are present in the continuous spectrum with this arrangement. Probably the limited resolving power of the grating has contributed to the interference phenomenon.

The two spectra in Fig. 3 showing no fringes have been obtained without the Wollaston prism unit, whereas the other two spectra have been taken with different settings of the prism, so that the maxima of the spectrum above have been replaced by minima in the spectrum below. An inspection of the Fraunhofer lines in these two spectra is of great interest. In fact, in the (broad) intensity maxima a single spectrum line appears as a doublet, whereas in the (broad) minima the same line appears as a broad and remarkably dark singlet. Already from this first oxperiment it seems evident that the effects predicted. by me are present, and that the new method of studying

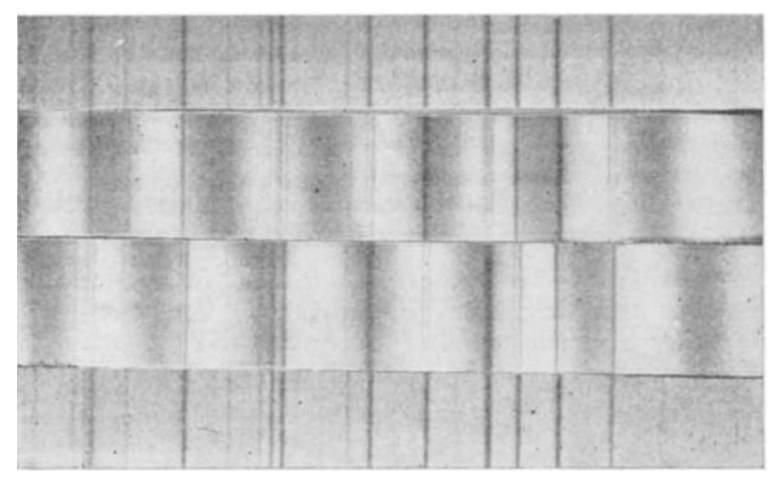

Fig. 3 interference may find interesting spectrographic applications.

However, only extensive experiments will prove if a real gain is obtained with the suggested method. We have to consider that there will be a certain reduction of light, even if the resolving power is increased. The use of 'heliometer lenses' or similar may prove better in this respect.

I thank Mr. U. Ericson and Mr. Y. Sundblad for valuable help with the preliminary work made in $1964^{2}$ and Dr. A. Wyller and Mr. U. Kusoffsky for assistance with the final spectrographic tests.

Swedish Solar Observatory,

YNGVE ÖHMAN * Anacapri.

* Present address: Stockholm Observatory, Saltsjöbaden, Sweden.

1 Öhman, Y., Astrophysica Norvegica, 9, 7 (1964).

2 Ohman, Y., Atti del Convegno sui campi magnetici solari, Roma 14-16, September 1964 (in the press). ${ }^{3}$ Ohman, Y., Applied Optics (in the press), see also E.S.R.O. Symp., Nice

${ }^{4}$ Unsöld, A., Phys, der Sternatmosphären, 252 (Berlin, 1955).

\section{The Temperature Scale}

Most of the points raised by Prof. J. C. Georgian on the subject of the temperature scale ${ }^{1}$ are discussed in Chapter 23 of $A$ Textbook of Heat by Allen and Maxwell (1948). The authors refer to a paper by Lewis and Adams ${ }^{2}$ making the very point about the dimensions of temperature, specific heat and enthalpy made by Prof. Georgian and proposing a temperature scale that would make $P V=n \theta$, where $n=$ Avogadro's number. They also discuss a proposal by Planck which seems to have been essentially that now made by Prof. Georgian.

There are, however, certain objections to these proposals. Unlike, for example, the Reynolds number, specific heat and enthalpy are dimensionless only so long as we bear in mind their relationship to water, for they rely for the invariance of their nominal numerical values on the retention of liquid water as the nominal datum. A change of a temperature scale from Kelvin to Georgian would therefore result merely in a change of the value of $J$ that had to be used in equations relating temperature and heat, unless we were prepared to re-calculate all our specific heat in terms of the relationship of the material to a perfect gas rather than to water. Furthermore, the unit of energy is nominally based on the properties of water (via the definition of the unit of mass), and to proceed to define temperature by invoking another substance altogether (perfect gas) is, I submit, unnecessary and undesirable.

I have already proposed ${ }^{3}$ that the calorie and the factor $J$ could be eliminated simply from temperature equations by the replacement of the Kelvin scale by one having $100 \times J$ degrees between the ice point and the steam point. I suggested ${ }^{3}$ that such a degree should be called the degree Joule. I think that the adoption of such a scale would be easior and more satisfactory than would the adoption of Prof. Georgian's scale, as it would not require any properties to be re-calculated, and I think it would commend itself more readily to practical users.

39 Park Road,

R. A. Laws

Watford, Hertfordshire.

I wish to thank Mr. Laws for refs. 2 and 3.

I was aware of ref. 2 and, in addition, Tolman ${ }^{4}$ has an interesting discussion of the dimensions of temperature. Only Tolman wishes to give temperature the units of $M L^{2} / T^{2}$, that is, allow the molecular mass number (molecular weight) to take on the dimension of mass. However, Porter ${ }^{5}$ in his book Method of Dimensions explains clearly that the dimension of temperature is indeod $L^{2} / T^{2}$. 